\title{
Effects of Tocols Rich Fraction Isolated from Rice Bran Oil Deodorizer Distillate on Plasma and Hepatic Lipid Concentrations in Rats
}

\author{
Tina A. Gomes ${ }^{1}$, Xuan Gu1ㅜ Cindi R. Brownmiller ${ }^{1}$, Luke Howard ${ }^{1}$, Cesar M. Compadre², \\ Sun-Ok Lee ${ }^{1^{*}}$ \\ ${ }^{1}$ Department of Food Science, University of Arkansas, Fayetteville, USA \\ ${ }^{2}$ Department of Pharmaceutical Science, University of Arkansas for Medical Science, Little Rock, USA \\ Email: "sunok@uark.edu
}

Received 24 August 2015; accepted 4 September 2015; published 7 September 2015

Copyright (C) 2015 by authors and Scientific Research Publishing Inc.

This work is licensed under the Creative Commons Attribution-NonCommercial International License (CC BY-NC).

http://creativecommons.org/licenses/by-nc/4.0/

(c) (1) (9) 0 pen Access

\begin{abstract}
A great deal of attention has been focused on the potential health benefits of using rice bran oil because it is a rich source of bioactive compounds. Rice bran oil deodorizer distillate (RBODD) is a byproduct obtained from distillation of rice bran oil. Elevated plasma cholesterol level is one of the major risk factors for coronary heart disease. The objective of this study was to investigate the effects of tocols (tocopherols + tocotrienols) rich fraction isolated from RBODD on plasma and liver lipid concentrations in rats. Male Sprague Dawley rats (6 weeks of age) were randomly assigned into three groups: normal fat control (NFC), high fat control (HFC) and high fat diet plus tocols rich fraction (RBODD). RBODD was administered daily for 3 weeks by oral gavage using $5 \%$ of Tween80 as a vehicle. The rats in the control groups received $5 \%$ of Tween-80 alone in the same manner. Blood samples and livers were collected at the end of the feeding period. RBODD group had significantly lower plasma triglyceride levels compared to the HFC group $(p<0.05)$. However, no significant changes for plasma total and lipoprotein cholesterol levels were found in RBODD compared with HFC. Compared to the rat fed HFC, hepatic free fatty acids were significantly reduced in the rats with the RBODD treatment $(p<0.05)$. The results suggest that the tocols rich fraction isolated from RBODD is associated with decreased plasma triglyceride and hepatic free fatty acids concentrations. Further study is needed to investigate the mechanism and optimal dose by which isomers of tocols lower triglyceride concentrations.
\end{abstract}

\section{Keywords}

Rice Bran Oil Deodorizer Distillate, Tocols, Oral Gavage, Triglycerides

\footnotetext{
${ }^{*}$ Corresponding author.
}

How to cite this paper: Gomes, T.A., Gu, X., Brownmiller, C.R., Howard, L., Compadre, C.M. and Lee, S.-O. (2015) Effects of Tocols Rich Fraction Isolated from Rice Bran Oil Deodorizer Distillate on Plasma and Hepatic Lipid Concentrations in Rats. Journal of Biosciences and Medicines, 3, 1-8. http://dx.doi.org/10.4236/jbm.2015.39001 


\section{Introduction}

Atherosclerotic cardiovascular diseases (CVDs), principally coronary heart disease and stroke, are a major cause of death in Western countries. Elevated blood cholesterol, total and low-density lipoprotein (LDL) cholesterol, or decreased high-density lipoprotein (HDL) cholesterol concentrations, are associated with the development of atherosclerotic cardiovascular diseases. Epidemiological studies have shown a direct link between CVDs and dietary component intake [1]. Diets high in vitamin E showed lower risks of cardiovascular diseases by downregulating cholesterol synthesis in the liver and reducing lipid peroxidation and platelet aggregation [2] [3]. In addition, vitamin E exerted the antioxidant activities against oxidative stress [4]. The recommended daily intake of vitamin $\mathrm{E}$ for adult is $15 \mathrm{mg} / \mathrm{day}$. Tocopherols (T) and tocotrienols (T3) are members of vitamin $\mathrm{E}$ family that occur in chromanol ring structure in plants and differ in the saturation of their side chains [5]. There are four isomers of each tocopherol and tocotrienol (alpha, beta, gamma and delta), named according to the position of the methyl group on the chromanol ring [6]. Tocopherols are present mainly in wheat, corn and soybeans, whereas tocotrienols are relatively abundant in palm oil, rice bran oil, oats, barley and anatto [7].

Rice bran oil (RBO) is the oil extracted from the germ and inner husk of rice and contains unsaturated linoleic and oleic fatty acids and bioactive compounds such as phytosterols, oryzanol, tocopherols andtocotrienols [8]. Due to its potential health-promoting properties, rice bran oil has received great interest in research. The hypocholesterolemic and anti-atherogenic effects of rice bran oil have shown in both animals [9]-[12] and humans [13] [14]. Studies have suggested that oryzanol is responsible for the beneficial effects of rice bran oil on plasma lipid concentrations [15] [16]. However, the refining steps (neutralization, bleaching, and deodorization) decrease the amounts of these bioactive compounds depending on the degree of processing and the origin of the rice bran. Several food technologies have been developed for successful extraction of these byproducts in order to utilize the beneficial nutritive values [17]. Improved distillation methods have been invented to extract RBO with stable lipase activity [18]. Rice bran oil deodorizer distillate (RBODD) is a valuable byproduct in the refinement of rice bran oil and has concentrated amounts of tocopherols, tocotrienols and other compounds. It is important to evaluate the biological properties of the tocols fraction in RBODD in order to develop a useful health product. The objectives of this study were to concentrate the tocols (tocopherols + tocotrientols) from rice bran oil deodorizer distillate (RBODD) and to evaluate the effect of tocol concentrate on plasma and liver lipid profiles in rats.

\section{Materials and Methods}

\subsection{Chemicals and Diets}

$\alpha$-, $\beta$-, $\gamma$ - and $\delta$-tocotrienols and $\alpha-, \beta-, \gamma$ - and $\delta$-tocopherols (94-95\% purity) purchased from Yasoo Health, Inc. (Johnson City, TN). All chemicals (analytical grade and HPLC grade) used to prepare buffers and mobile phases were purchased from VWR international Inc. (Suwanee, GA). Rice bran oil deodorizer distillate (RBODD) was generously provided by Riceland Foods (Jonesboro, AR). All dietary ingredients were purchased from Harlan Laboratories Inc. (Indianapolis, IN).

\subsection{Extraction of RBODD}

Rice bran oil deodorizer distillate (RBODD) was extracted to concentrate the tocols (tocopherols + tocotrienols). Tocols were concentrated using the method of Ko et al. [18] with some modifications. Stock samples of $5 \mathrm{~g}$ RBODD were mixed with $50 \mathrm{ml}$ acetonitrile in a 250-ml flat-bottom flask. The flask was fitted with a reflux condenser and the mixture was brought to boiling then refluxed on a hot plate for $30 \mathrm{~min}$ to destroy volatiles and aggregative residues. The mixture was cooled to ambient temperature and stored for 24 hours at $-20^{\circ} \mathrm{C}$ in order to precipitate the cold insoluble sterols from the soluble tocols. The liquid fraction was separated from the insoluble residue by centrifuge at 10,000 rpm for $10 \mathrm{~min}$. The acetonitrile was evaporated from the liquid fraction using a Speed Vac concentrator (Thermo Savant, Holbrook, NY). The remaining substance was mixed with $4 \mathrm{ml}$ of $5 \%(\mathrm{w} / \mathrm{v})$ pyrogallol in ethanol and $30 \mathrm{ml}$ of ethanol in a 120-ml flat-bottom flask along with a stir bar. The flask was fitted with a reflux condenser and the mixture heated to boiling. Once boiling, the condenser was removed and $1 \mathrm{ml}$ of $50 \%(\mathrm{w} / \mathrm{v})$ aqueous potassium hydroxide solution was added to the mixture. The tocols were saponified at $70^{\circ} \mathrm{C}$ for 30 min with constant stirring. The flask was placed in an ice bath to stop the reaction. The mixture was transferred to a 500-ml separatory funnel, and $30 \mathrm{ml}$ diethyl ether and $20 \mathrm{ml}$ distilled water were 
added. The diethyl ether extraction was repeated two times and the ether fractions were pooled. The pooled diethyl ether was washed three times with $20 \mathrm{ml}$ distilled water, and then filtered through anhydrous sodium sulfate for $30 \mathrm{~min}$ to remove any excess water. The diethyl ether was evaporated using the Speed Vac concentrator. Extracts were resolubilized with hexane to a volume to $5 \mathrm{ml}$ and filtered through a $0.45 \mu \mathrm{m}$ Millipore membrane before injection into the HPLC (High-performance liquid chromatography).

\subsection{Tocol Identification and Quantification}

Tocols were identified and quantified using a Waters Alliance 2690 separations module, a Waters 474 fluorescence detector, and Empower Pro 2 Software (Waters Corp., Milford, MA). Separation was performed using a Phenomenex Luna, $250 \times 4.6 \mathrm{~mm}$ column and an isocratic mobile phase of hexane/ethyl acetate/acetic acid 97.3:1.8:0.9 $(\mathrm{v} / \mathrm{v} / \mathrm{v})$ at $1 \mathrm{ml} / \mathrm{min}$. Peaks were detected at an excitation wavelength of $294 \mathrm{~nm}$ and an emission wavelength of $326 \mathrm{~nm}$. Unknowns were compared to known standards of $\alpha-, \beta$-, $\gamma$ - and $\delta$-tocotrienols and $\alpha-, \beta$-, $\gamma$ - and $\delta$-tocopherols.

\subsection{Animals and Experimental Design}

All experimental protocols were reviewed and approved by the Institutional Animal Care and Use Committee (IACUC) of the University of Arkansas. Thirty-three $(\mathrm{n}=33)$ Sprague Dawley male rats were purchased from Harlan Laboratories Inc. (Indianapolis, IN), at 6-week of age. They were group housed (two per cage) in a temperature-controlled room $\left(23^{\circ} \mathrm{C}\right)$ maintained on a 12-h light: dark cycle. The rats were allowed to acclimate to their new environment for one week and initial body weights were recorded prior to acclimatization period. After acclimatization, the rats were switched to AIN93G diet with appropriate fat content for the study group. The rats, with the same average body weight in each group, were randomly divided into 3 groups: normal fat control (NFC, $n=9$ ), high fat control (HFC, $n=12$ ) and HFC plus tocols rich fraction isolated from RBODD (RBODD, $\mathrm{n}=12$ ). NFC group was fed a diet containing $10 \%$ of energy from fat, whereas both HFC and RBODD received diet with $38 \%$ of energy from fat. The rats were weighed weekly for the 3-week study period. The feed was replenished every three days. Any spill or waste of feed was recorded with the data. After the week of acclimatization, the rats in RBODD group were treated with $45 \mathrm{mg}$ of tocols rich fraction isolated from rice bran oil deodorizer distillate in $0.5 \mathrm{~mL}$ of $5 \%$ Tween-80 solution; administered via gavage per animal per day (around 8am). The animals in group NFC and HFC received $0.5 \mathrm{~mL}$ of $5 \%$ Tween- 80 solution in the same manner as the RBODD group. In order to randomize administration and minimize discrepancy during gavage, all cages were rotated in sequence for each day of treatment. At the end of the 3-week treatment period, the rats were fasted for 12 hours and weighed before euthanization in $\mathrm{CO}_{2}$ chamber. Maximum amount of blood was drawn via cardiac puncture using a syringe coated with anti-coagulant EDTA (ethylenediaminetetraacetic acid). Plasma was collected via centrifugation at $1000 \mathrm{~g}$ for 10 minutes at $4^{\circ} \mathrm{C}$. Major organs such as the liver, cecum, and cecum content were extracted to be stored in liquid nitrogen for further analysis.

\subsection{Plasma and Liver Lipid Analysis}

Total cholesterol, triglyceride and HDL-cholesterol levels in plasma were quantified using fluorometric and colorimetric assays. Cayman kits were used for total cholesterol and triglyceride assays; and Sigma Aldrich was used for HDL-cholesterol quantification in plasma. Total liver cholesterol (Sigma Aldrich), triglycerides (Biovsion) and free fatty acids (Sigma Aldrich) were measured by homogenizing all lobes of liver using protocols provided in the kits.

\subsection{Statistical Analysis}

Statistical software SAS (version 9.4) was used for all statistical analyses. The results were analyzed by using one-way analysis of variance (ANOVA). Data of initial body weight, weight gain, and food consumption were presented as mean \pm standard deviation (SD). Other values were expressed as mean \pm standard error mean (SEM) and statistical significance was set at $\mathrm{p}<0.05$.

\section{Results}

Table 1 showed the tocol composition (tocopherols + tocotrienols) of the RBODD after the extraction process 
and the HPLC chromatography results were shown in Figure 1. The tocol concentrations increased from 4\% tocols in the RBODD (starting material) to $34 \%$ tocols after the extraction process. The major tocols were $\delta$-tocopherol and $\gamma$-tocotrienol.

There were no significant differences between dietary treatments for initial body weight, weight gain, and food consumption (Table 2). The average liver weight for NFC, HFC, and RBODD groups was $10.2 \pm 0.3 \mathrm{~g}$, $11.1 \pm 0.5 \mathrm{~g}$ and $11.1 \pm 0.3 \mathrm{~g}$, respectively. No significant differences of liver weight among groups were found.

Table 3 showed plasma and hepatic lipid profiles in rats after the three experimental treatments. Plasma total cholesterol and HDL cholesterol were not significantly different in the rats fed the RBODD treatment compared to the HFC-fed rats (Table 3). Plasma triglyceride concentration was significantly lower in the RBODD group compared to the NFC and HFC groups $(\mathrm{p}<0.05)$.

Compared to the HFC-fed rats, hepatic total cholesterol and triglyceride concentrations were not significantly different in the rats fed the RBODD (Table 3). However, RBODD treatment had a slightly higher level of he patic cholesterol as compared to the HFC group. Hepatic free fatty acids were significantly lower in the NFC

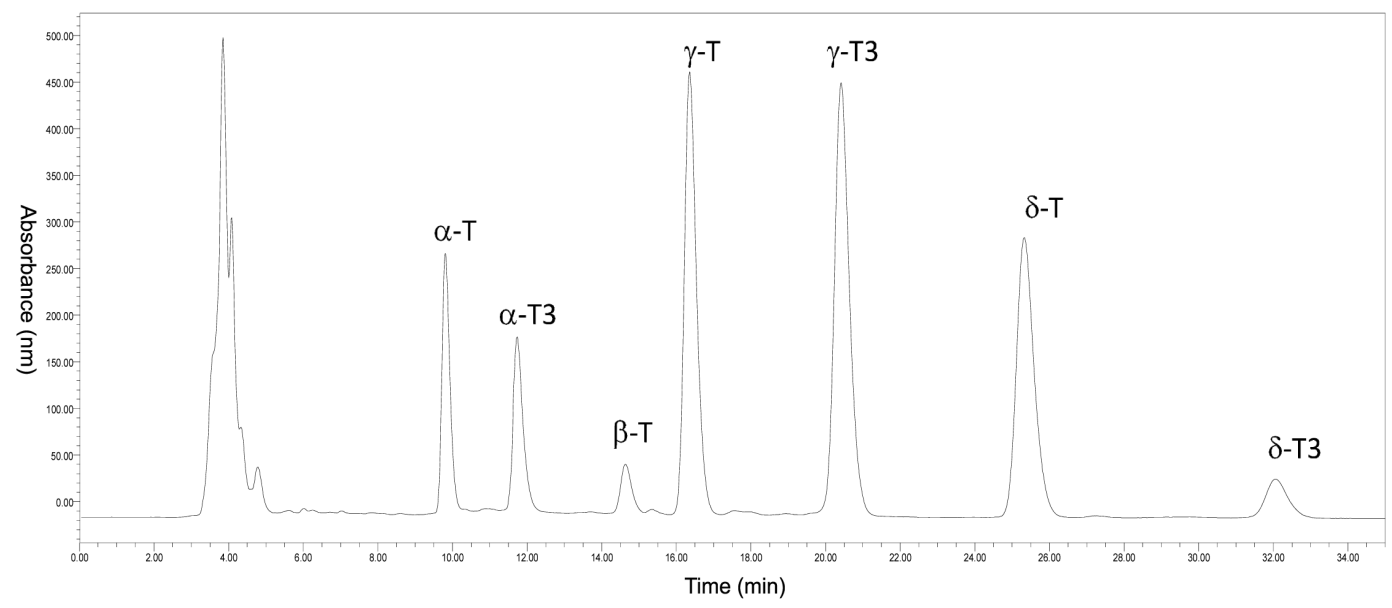

Figure 1. HPLC chromatogram of $\alpha-, \beta$-, $\gamma$ - and $\delta$-tocopherols (T) and $\alpha-, \beta$-, $\gamma$ - and $\delta$-tocotrienols (T3) in RBODD.

Table 1. Tocol composition of the fraction isolated from rice bran oil deodorizer distillate.

\begin{tabular}{|c|c|c|}
\hline \multirow{2}{*}{ Isomers } & \multicolumn{2}{|c|}{ Tocols $(\mathrm{mg} / \mathrm{g})^{1}$} \\
\hline & Tocopherols (T) & Tocotrienols (T3) \\
\hline Alpha & $56.3 \pm 0.3$ & $15.1 \pm 0.1$ \\
\hline Beta & $8.3 \pm 0.1$ & $\mathrm{ND}^{2}$ \\
\hline Gamma & $60.9 \pm 0.1$ & $121.1 \pm 0.3$ \\
\hline Delta & $65.8 \pm 0.2$ & $14.6 \pm 0.1$ \\
\hline Total & $191.3 \pm 0.2$ & $150.8 \pm 0.2$ \\
\hline
\end{tabular}

Values are expressed as mean $\pm \mathrm{SD} .{ }^{1}$ Amount $(\mathrm{mg})$ of each isomer present in one gram of RBODD. ${ }^{2}$ ND: No data available.

Table 2. Initial body weight, body weight gain and food consumption of rats.

\begin{tabular}{ccccc}
\hline Groups & N & Initial body weight $(\mathbf{g})$ & Body weight gain $(\mathbf{g})$ & Food consumption $(\mathrm{g} / \mathbf{d})$ \\
\hline NFC & 9 & $203.0 \pm 9.5$ & $85.4 \pm 19.7$ & $19.5 \pm 2.8$ \\
HFC & 12 & $203.0 \pm 11.2$ & $97.9 . \pm 21.4$ & $17.0 \pm 2.7$ \\
RBODD & 12 & $203.0 \pm 11.0$ & $102.1 \pm 15.4$ & $17.1 \pm 1.7$ \\
\hline
\end{tabular}

Values are expressed as mean \pm SD. NFC: normal fat control group; HFC: high fat control group; RBODD: high fat diet \pm rice bran oil deodorizer distillate treatment. 
Table 3. Plasma and liver lipids and lipoprotein concentrations in rats.

\begin{tabular}{cccc}
\hline & \multicolumn{1}{c}{ Groups } & \\
\cline { 2 - 3 } & NFC $(\mathbf{n}=\mathbf{9})$ & HFC $(\mathbf{n}=\mathbf{1 2})$ & RBODD $(\mathbf{n}=\mathbf{1 2})$ \\
\hline Plasma (mmol/L) & & & $1.78 \pm 0.06^{\mathrm{a}}$ \\
Total cholesterol & $1.51 \pm 0.03^{\mathrm{b}}$ & $1.68 \pm 0.05^{\mathrm{a}}$ & $1.25 \pm 0.07^{\mathrm{b}}$ \\
HDL cholesterol & $1.41 \pm 0.08^{\mathrm{a}}$ & $1.22 \pm 0.06^{\mathrm{b}}$ & $0.43 \pm 0.02^{\mathrm{b}}$ \\
Triglycerides & $0.53 \pm 0.04^{\mathrm{a}}$ & $0.52 \pm 0.03^{\mathrm{a}}$ & $4.2 \pm 0.2^{\mathrm{a}}$ \\
Liver (mg/g tissue) & & & $1.9 \pm 0.1^{\mathrm{a}}$ \\
Total cholesterol & $3.4 \pm 0.3^{\mathrm{b}}$ & $3.8 \pm 0.3^{\mathrm{a}}$ & $3.6 \pm 0.7^{\mathrm{b}}$ \\
Triglycerides & $1.6 \pm 0.2^{\mathrm{b}}$ & $1.9 \pm 0.1^{\mathrm{a}}$ & $6.0 \pm 0.9^{\mathrm{a}}$ \\
Free fatty acids & $3.7 \pm 1.0^{\mathrm{b}}$ & & \\
\hline
\end{tabular}

Values are expressed as mean \pm SEM. Superscripts not sharing a common letter within the same row are significantly different among groups at $\mathrm{p}<$ 0.05. NFC: normal fat control group; HFC: high fat control group; RBODD: high fat diet \pm rice bran oil deodorizer distillate treatment.

and RBODD groups compared to the HFC group $(\mathrm{p}<0.05)$.

\section{Discussion}

Elevated levels of plasma triglycerides are considered an indication for increased risk of cardiovascular diseases. Some triglyceride-rich lipoproteins (TRLs) can promote atherogenesis independently from LDL. Remnant species produced by partial hydrolysis of TRLs may attach cholesterol esters from HDL [19]. These remnant species can easily accumulate endothelial and be form foam cells with macrophage and contribute to the formation of atherosclerotic plaque [20].

In this present study, we found a reduction of plasma triglycerides as an effect of RBODD treatment $(8.5 \mathrm{mg}$ tocopherols and $6.7 \mathrm{mg}$ tocotrienols per day) when compared to each control group. In addition, the RBODD treatment lowered hepatic free fatty acid (FFA) concentrations and high fat control (HFC) showed an increase of FFA levels in relative to normal control (NFC) and RBODD. Our results agree with the findings that diabetic rats fed the rice bran oil diet (60 - $90 \mathrm{mg} \gamma$-tocotrienol and 35.2 - 52.8 $\mathrm{g} \gamma$-oryzanol per $100 \mathrm{~g}$ diet) had lower plasma triglyceride and LDL cholesterol levels than rats fed the control diet after 4 weeks of treatment [21]. In the present study,the high fat control group did not show a comparable higher triglyceride levels in comparison to normal control group. A potential explanation to this result may be contributed to the feeding uptake/behavior of each rat and the time of each treatment. Rodent being nocturnal animals may show different metabolic patterns when treated in the morning versus evening. The stress of disrupting their sleep cycle during day may also interfere with their metabolic rate.

Although the majority of the literature showed that the plasma lowering associated with rice bran oil occurs mainly in the total and LDL cholesterol, our results showed the lack of effect of RBODD on plasma total cholesterol. The rats fed the RBODD and HFC were not significantly different from each other with regard to plasma and hepatic total cholesterol levels. This finding is in agreement with previous studies that there are no positive reduction of plasma and hepatic cholesterol concentrations [21]-[24]. Chou et al. [25] observed that the rice bran oil group ( $0.9 \mathrm{~g} \gamma$-tocotrienol and $5.25 \mathrm{~g} \gamma$-oryzanol $/ \mathrm{kg}$ diet) did not lower plasma total cholesterol, triglyceride, and LDL cholesterol and hepatic triglyceride levels compared with the control group.

Tocotrienols are relatively abundant in rice bran oil, palm oil, oatsand barley. Numerous studies reported that the effects of tocotrienols and tocotrienol-rich fractions on lipid metabolism in animals [26]-[29] and humans [30]-[33]. The mechanism of the hypocholesterolemic effect of tocotrienols was revealed as the suppression of 3-Hydroxy-3-methylglutaryl-coenzyme A (HMG-CoA) reductase activity and inhibition of expression of HMGCoA reductase mRNA [21] [34]. However, tocopherols and tocotrienols have been reported to influence the cholesterol metabolism in different manners. Qureshi et al. [35] reported that $\alpha$-tocopherol reduced the inhibitory effects of $\gamma$-tocotrienol on HMG-CoA activity. $\alpha$-tocopherol induced HMG-CoA reductase activity, while tocotrienol decreased HMG-CoA reductase activity [34] [36]. Except $\alpha$-tocopherol, it is still not clear the interaction between different isomers of tocopherols and tocotrienols. Therefore, further studies can be in the direction of investigating the potential mechanisms of each purified tocopherols and tocotrienols or combination of tocols 
to lower plasma lipid concentrations, and examining the effects of different dose of each tocols in reducing plasma lipid levels.

\section{Conclusion}

The current study suggests that the tocols (tocopherols + tocotrienols) rich fraction from RBODD has an effect on lowering plasma triglyceride and hepatic free fatty acid levels in Sprague Dawley rats. In order to validate the hypotriglyceridemic effects of the tocols rich fraction in RBODD, further study is needed to define the mechanism and which isomers of tocols are responsible for its triglyceride-lowering effects.

\section{Acknowledgements}

Funding provided by the Arkansas Bioscience Institution is gratefully acknowledged.

\section{References}

[1] Krehl, W. (1997) The Nutritional Epidemiology of Cardiovascular Disease. Annals of the New York Academy of Sciences, 300, 335-359. http://dx.doi.org/10.1111/j.1749-6632.1977.tb19333.x

[2] Landrier, J.F., Gouranton, E., Reboul, E., Cardinault, N., El Yazidi, C. and Malezet-Desmoulins, C. (2010) Vitamin E Decreases Endogenous Cholesterol Synthesis and Apo-AI-Mediated Cholesterol Secretion in Caco-2 Cells. Journal of Nutritional Biochemistry, 21, 1207-1213. http://dx.doi.org/10.1016/j.jnutbio.2009.10.008

[3] Kaul, N., Devaraj, S. and Jialal, I. (2001) Alpha-Tocopherol and Atherosclerosis. Experimental Biology and Medicine, 226, 5-12.

[4] Yoshida, Y., Niki, E. and Noguchi, N. (2003) Comparative Study on the Action of Tocopherols and Tocotrienols as Antioxidant: Chemical and Physical Effects. Chemistry and Physics of Lipids, 123, 63-75. http://dx.doi.org/10.1016/S0009-3084(02)00164-0

[5] Horvath, G., Wessjohann, L., Bigirimana, J., Jansen, M., Guisez, Y., Caubergs, R. and Horemans, N. (2006) Differential Distribution of Tocopherols and Tocotrienols in Photosynthetic and Non-Photosynthetic Tissues. Phytochemistry, 67, 1185-1195. http://dx.doi.org/10.1016/j.phytochem.2006.04.004

[6] Sen, C.K., Khanna, S. and Roy, S. (2006) Tocotrienols: Vitamin E beyond Tocopherols. Life Sciences, 78, $2088-2098$. http://dx.doi.org/10.1016/j.lfs.2005.12.001

[7] Munné-Bosch, S. and Alegre, L. (2002) The Function of Tocopherols and Tocotrienols in Plants. Critical Reviews in Plant Sciences, 21, 31-57. http://dx.doi.org/10.1080/0735-260291044179

[8] Ghosh, M. (2007) Review on Recent Trends in Rice Bran Oil Processing. Journal of the American Oil Chemists' Society, 84, 315-324. http://dx.doi.org/10.1007/s11746-007-1047-3

[9] Kahlon, T.S., Saunders, R.M., Sayre, R.N., Chow, F.I., Chiu, M.M. and Betschart, A.A. (1992) Cholesterol-Lowering Effects of Rice Bran and Rice Bran Oil Fractions in Hypercholesterolemic Hamsters. Cereal Chemistry, 69, 485-489.

[10] Ausman, L.M., Rong, N. and Nicolosi, R.J. (2005) Hypocholesterolemic Effect of Physically Refined Rice Bran Oil: Studies of Cholesterol Metabolism and Early Atherosclerosis in Hypercholesterolemic Hamsters. Journal of Nutritional Biochemistry, 16, 521-529. http://dx.doi.org/10.1016/j.jnutbio.2005.01.012

[11] Kahlon, T.S., Chow, F.I., Chiu, M.M., Hudson, C.A. and Sayre, R.N. (1996) Cholesterol-Lowering by Rice Bran and Rice Bran Oil Unsaponifiable Matter in Hamsters. Cereal Chemistry, 73, 69-74.

[12] Nicolosi, R.J. Ausman, L.M. and Hegsted, D.M. (1991) Rice Bran Oil Lowers Serum Total and Low Density Lipoprotein Cholesterol and Apo B Levels in Nonhuman Primates. Atherosclerosis, 88, 133-142. http://dx.doi.org/10.1016/0021-9150(91)90075-E

[13] Raghuram, T.C., Rao, U.B. and Rukmini, C. (1989) Studies on Hypolipidemic Effects of Dietary Rice Bran Oil in Human Subjects. Nutrition Reports International, 39, 889-895.

[14] Lichtenstein, A.H., Ausman, L.M., Carrasco, W., Gualtieri, L.J., Jenner, J.L., Ordovas, J.M., Nicolosi, R.J., Goldin, B.R. and Schaefer, E.J. (1994) Rice Bran Oil Consumption and Plasma Lipid Levels in Moderately Hypercholesterolemic Humans. Arteriosclerosis, Thrombosis, and Vascular Biology, 14, 549-556. http://dx.doi.org/10.1161/01.ATV.14.4.549

[15] Wilson, T.A., Nicolosi, R.J., Woolfrey, B. and Kritchevsky, D. (2007) Rice Bran Oil and Oryzanol Reduce Plasma Lipid and Lipoprotein Cholesterol Concentrations and Aortic Cholesterol Ester Accumulation to a Greater Extent than Ferulic Acid in Hypercholesterolemic Hamsters. The Journal of Nutritional Biochemistry, 18, 105-112. http://dx.doi.org/10.1016/j.jnutbio.2006.03.006 
[16] Chandrashelkar, P., Kumar, P.K., Ramesh, H.P., Lokesh, B.R. and Krishna, A.G. (2014) Hypolipidemic Effect of Oryzanol Concentrate and Low Temperature Extracted Crude Rice Bran Oil in Experimental Male Wistar Rats. Journal of Food Science and Technology, 51, 1279-1285.

[17] Van Hoed, V., Depaemelaere, G., Ayala, J.V., Santiwattana, P., Verhé, R. and De Greyt, W. (2006) Influence of Chemical Refining on the Major and Minor Components of Rice Brain Oil. Journal of the American Oil Chemists' Society, 84, 315-324. http://dx.doi.org/10.1007/s11746-006-1206-y

[18] Ko, S-N., Lee, S-M. and Kim, I-H. (2008) The Concentration of Tocols from Rice Bran Oil Deodorizer Distillate Using Solvent. European Journal of Lipid Science and Technology, 110, 914-919. http://dx.doi.org/10.1002/ejlt.200700310

[19] Brewer, H. (1999) Hypertriglyceridemia: Changes in the Plasma Lipoproteins Associated with an Increased Risk of Cardiovascular Disease. The American Journal of Cardiology, 83, 3F-12F. http://dx.doi.org/10.1016/S0002-9149(99)00308-2

[20] Botham, K.M., Napolitano, M. and Bravo, E. (2010) Induction of Macrophage Monocyte Chemoattractant Secretion by Triacylglycerol Rich Lipoproteins. Atherosclerosis Supplements, 11, 70-71. http://dx.doi.org/10.1016/j.atherosclerosissup.2010.04.018

[21] Chen, C.W. and Cheng, H.H. (2006) A Rice Bran Oil Diet Increases LDL-Receptor and HMG-CoA Reductase mRNA Expressions and Insulin Sensitivity in Rats with Streptozotocin/Nicotinamide-Induced Type 2 Diabetes. Journal of Nutrition, 136, 1472-1476.

[22] Wahlqvist, M.L., Krivokuca-Bogetic, Z., Lo, C.S., Hage, B., Smith, R. and Likito, W. (1992) Differential Serum Responses of Tocopherols and Tocotrienols during Vitamin Supplementation in Hypercholesterolaemic Individuals without Change in Coronary Risk Factors. Nutrition Research, 12, S181-S201. http://dx.doi.org/10.1016/S0271-5317(05)80463-4

[23] Stampfer, M.J., Willet, W., Castelli, W.P., Taylor, J.O., Fine, J. and Hennekens, C.H. (1983) Effect of Vitamin E on Lipids. The American Journal of Clinical Nutrition, 79, 714-716.

[24] Kesaniemi, Y.A. and Grundy, S.M. (1982) Lack of Effect of Tocopherol on Plasma Lipids and Lipoproteins in Man. The American Journal of Clinical Nutrition, 36, 224-228

[25] Chou, T.W., Ma, C.Y., Cheng, H.H., Chen, Y.Y. and Lai, M.H. (2009) A Rice Bran Oil Diet Improves Lipid Abnormalities and Suppress Hyperinsulinemic Responses in Rats with Streptozotocin/Nicotinamide-Induced Type 2 Diabetes. Journal of Clinical Biochemistry and Nutrition, 45, 29-36. http://dx.doi.org/10.3164/jcbn.08-257

[26] Qureshi, A.A., Qureshi, N., Haslerrapacz, J.O., Weber, F.E., Chaudhary, V., Crenshaw, T.D., Gapor, A., Ong A.S.H and Chong, Y.H. (1991) Dietary Tocotrienols Reduce Concentrations of Plasma Cholesterol, Apolipoprotein B, Thromboxane B2, and Platelet Factor 4 in Pigs with Inherited Hyperlipidemias. The American Journal of Clinical Nutrition, 53, S1042-S1046.

[27] Qureshi, A.A. and Peterson, D.M. (2001) The Combined Effects of Novel Tocotrienols and Lovastatin on Lipid Metabolism in Chickens. Atherosclerosis, 156, 39-47. http://dx.doi.org/10.1016/S0021-9150(00)00612-2

[28] Qureshi, A.A., Peterson, D.M., Hasler-Rapacz, J.O. and Rapacz, J. (2001) Novel Tocotrienols of Rice Bran Suppress Cholesterogenesis in Hereditary Hypercholesterolemic Swine. Journal of Nutrition, 131, 223-230.

[29] Raederstorff, D., Elste, V., Aebischer, C. and Weber, P. (2002) Effect of Either Gamma-Tocotrienol or a Tocotrienol Mixture on the Plasma Lipid Profile in Hamsters. Annals of Nutrition and Metabolism, 46, 17-23. http://dx.doi.org/10.1159/000046748

[30] Tan, D.T., Khor, H.T., Low, W.H., Ali, A. and Gapor, A. (1991) Effect of a Palm-Oil-Vitamin E Concentrate on the Serum and Lipoprotein Lipids in Humans. The American Journal of Clinical Nutrition, 53, 1027S-1030S.

[31] Qureshi, A.A., Bradlow, B.A., Brace, L., Manganello, J., Peterson, D.M., Pearce, B.C., Wright, J.J., Gapor, A. and Elson, C.E. (1995) Response of Hypercholesterolemic Subjects to Administration of Tocotrienols. Lipids, 30, 1171-1177. http://dx.doi.org/10.1007/BF02536620

[32] Qureshi, A.A., Sami, S.A., Salser, W.A. and Khan, F.A. (2002) Dose-Dependent Suppression of Serum Cholesterol by Tocotrienol-Rich Fraction (TRF25) of Rice Bran in Hypercholesterolemic Humans. Atherosclerosis, 161, 199-207. http://dx.doi.org/10.1016/S0021-9150(01)00619-0

[33] Qureshi, A.A., Qureshi, N., Wright, J.J.K., Shen, Z., Kramer, G., Gapor, A., Chong, Y.H., DeWitt, G., Ong, A.S.H., Peterson, D.M. and Bradlow, B.A. (1991) Lowering of Serum-Cholesterol in Hypercholesterolemic Humans by Tocotrienols (Palmvitee). The American Journal of Clinical Nutrition, 53, S1021-S1026.

[34] Parker, R.A., Pearce, B.C., Clark, R.W., Gordon, D.A. and Wright, J.J.K. (1993) Tocotrienols Regulate Cholesterol Production in Mammalian Cells by Post-Transcriptional Suppression of 3-Hydroxy-3-Methylglutaryl-Coenzyme A Reductase. The Journal of Biological Chemistry, 268, 11230-11238.

[35] Qureshi, A.A., Pearce, B.C., Nor, R.M., Gapor, A., Peterson, D.M. and Elson, C.E. (1996) Dietary Alpha-Tocopherol 
Attenuates the Impact of Gamma-Tocotrienol on Hepatic 3-Hydroxy-3-Methylglutaryl Coenzyme A Reductase Activity in Chickens. Journal of Nutrition, 126, 389-394.

[36] Pearce, B.C., Parker, R.A., Deason, M.E., Qureshi, A.A. and Wright, J.J.K. (1992) Hypocholesterolemic Activity of Synthetic and Natural Tocotrienols. Journal of Medicinal Chemistry, 35, 3595-3606.

http://dx.doi.org/10.1021/jm00098a002 